Molecules 2005, 10, 822-832

\title{
molecules
}

ISSN 1420-3049

http://www.mdpi.org

\section{Synthesis of 1,3,4-Thiadiazole, 1,3,4-Thiadiazine, 1,3,6-Thia- diazepane and Quinoxaline Derivatives from Symmetrical Dithiobiureas and Thioureidoethylthiourea Derivatives}

\author{
Alaa A. Hassan*, Aboul-Fetouh E. Mourad, Kamal M. El-Shaieb and Ashraf H. Abou-Zied \\ Chemistry Department, Faculty of Science, El-Minia University, El-Minia, A. R. Egypt
}

Dedicated to Professor Dietrich Döpp on the occasion of his $65^{\text {th }}$ birthday

* Author to whom correspondence should be addressed, e-mail: alaahassan2001@yahoo.com

Received: 8 March 2004 / Accepted: 12 April 2004 / Published: 31 August 2005

\begin{abstract}
Reactions of $N, N^{`}$-disubstituted hydrazinecarbothioamides 8a-c and substituted thioureidoethylthioureas 9a-c with 2,3,5,6-tetrachloro-1,4-benzoquinone (chloranil, 10a) and 2,3,5,6-tetrabromo-1,4-benzoquinone (bromanil, 10b) to form $N, N^{`}$-disubstituted $[1,3,4]$ thiadiazole-2,5-diamines 11a-c, 6,7-dichloro-3-substituted amino-1H-benzo[1,3,4]thiadiazine-5,8-diones 12a-c, 2,3,7,8-tetrahalothianthrene-1,4,6,9-tetraones 13a,b, 5,6,8trihalo-7-oxo-3,7-dihydro-2H-quinoxaline-1-carbothioic acid substituted amides 14a-c, 15a-c and 7-substituted imino-[1,3,6]thiadiazepane-3-thiones 16a-c are reported. Rationales for the observed conversions are presented.
\end{abstract}

Keywords: Tetrahalo-1,4-benzoquinones; Cyclocondensation; Heterocyclic compounds.

\section{Introduction}

Addition of nitrogen nucleophiles to benzo-, and naphthoquinones represents a common synthetic route to many dyestuffs and medicinals [1-13]. The reactions of 2,3-dichloro-1,4-naphthoquinone (1) with thioacetamide or with thiourea to give 2-methyl- and 2-aminonaphtho[2,3-d]thiazole-4,9-diones 3 and $\mathbf{4}$, as well as the synthesis of bisthiazole 7 from $\mathbf{1}$ and dithiooxamide were first reported by Hammam et al. [14]. They also claimed that the intermediates, 2-thioamido-3-chloro-1,4-naphthoquinones $\mathbf{5}$ and $\mathbf{6}$ could be isolated from the reaction medium and separately transformed into thiazoles 
by boiling in aqueous ethanol containing sodium bicarbonate. Later, this work was repeated by Katritzky et al. $[15,16]$ and, in agreement with the earlier results, they found that $\mathbf{1}$ reacted with a variety of thioamides in dimethylformamide or in dimethylsulfoxide in the presence of triethylamine yielding the corresponding thiazoles $\mathbf{3}$ and $\mathbf{4}$ and with dithiooxamides to form the bisthiazole $\mathbf{7}$.

Matsuoka and co-workers [17-19] subsequently claimed that the previous work was in error and the reactions of $\mathbf{1}$ with thioacetamide, thiourea and dithiooxamide all gave the same product, namely dibenzo[b,i] thianthrene-5,7,12,14-tetraone (2) but not the thiazoles 3, 4 and 7.

Scheme 1

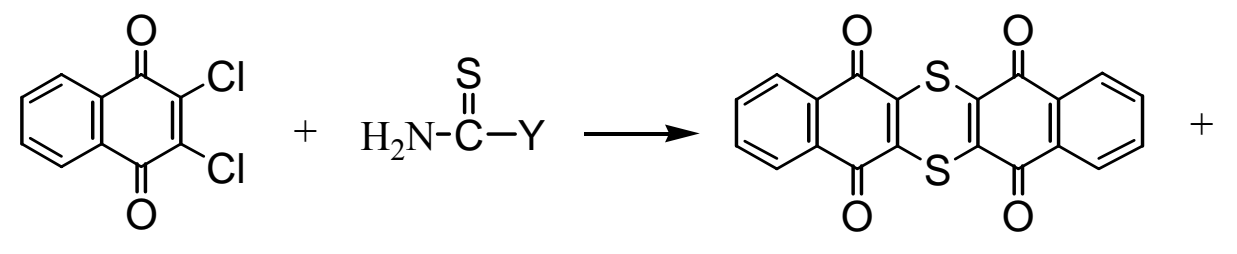<smiles>[Y]c1nc2c(s1)C(=O)c1ccccc1C2=O</smiles><smiles>[Y]C(=S)NC1=C(Cl)C(=O)c2ccccc2C1=O</smiles>

$$
\begin{array}{ll}
\text { 3: } \mathrm{Y}=\mathrm{CH}_{3} & \text { 5: } \mathrm{Y}=\mathrm{CH}_{3} \\
\text { 4: } \mathrm{Y}=\mathrm{NH}_{2} & \text { 6: } \mathrm{Y}=\mathrm{NH}_{2}
\end{array}
$$<smiles>O=C1c2ccccc2C(=O)c2sc(-c3nc4c(s3)C(=O)c3ccccc3C4=O)nc21</smiles>

In view of these discrepancies, Katritzky and co-workers subsequently reexamined some of those reactions [20]. Although the product reported to have been isolated by Matsuoka et al. was indeed formed, in all cases the 1,4-dithiine was accompanied by the corresponding 1,3-thiazole, although in some cases product separation was difficult.

Several authors have investigated the heterocyclization of 1,6-disubstituted dithiobiureas in basic or acidic media [21-28]. We report herein the results of our recent investigations on the reactions of symmetrical dithiobiureas as well as thioureidoethylthiourea derivatives with both chloranil (10a) and bromanil (10b).

\section{Results and Discussion}

On adding tetrahydrofuran (THF) solutions of 8a-c to 2:1 solutions of 10a,b in the same solvent, appearance of a green colour which gradually changed to blue was observed. When the reaction was monitored spectrophotometrically (at $10{ }^{\circ} \mathrm{C}$ ), an absorption maximum was observed in the visible region at 536-508 $\mathrm{nm}$ that was assigned to the formation of an unstable charge-transfer complex (CTC), since neither the thiourea derivatives 8a-c nor 10a,b absorb alone in this region. After standing for 48 
hours at room temperature, 2,3,7,8-tetrahalothianthrene-1,4,6,9-tetraones 13a,b were precipitated as the major products (41-44\%). From the filtrate the substituted amino-6,7-dichloro-benzo[1,3,4]thiadiazine-5,8-diones 12a-c (22-28\%), together with 2,5-disubstituted amino-1,3,4-thiadiazoles 11a-c $(12-15 \%$ in case of $\mathbf{1 0 a}, 21-26 \%)$ in case of $\mathbf{1 0 b})$, were isolated as minor products by preparative thin layer chromatography.

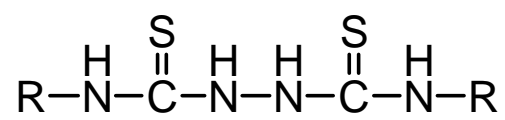

8<smiles>[X]C1=C([X])C(=O)C([X])=C([X])C1=O</smiles>

10: $\mathbf{a}, \mathrm{X}=\mathrm{Cl}$

b, $\mathrm{X}=\mathrm{Br}$<smiles></smiles>

13: $\mathbf{a}, \mathrm{X}=\mathrm{Cl}$

b, $\mathrm{X}=\mathrm{Br}$<smiles>[R]Nc1nnc(N[R])s1</smiles>

11<smiles>[R]NC(=S)N1CCN=C2C([X])=C([X])C(=O)C([X])=C21</smiles>

14: $\mathrm{X}=\mathrm{Cl}$

15: $\mathrm{X}=\mathrm{Br}$

8, 9, 11-12, 14-16: a, $\mathrm{R}=\mathrm{Ph} ; \mathbf{b}, \mathrm{R}=\mathrm{PhCH}_{2} ; \mathbf{c}, \mathrm{R}=$ allyl

As an example, the structural assignment of 12a was supported by the following spectral data: in its ${ }^{13} \mathrm{C}$-NMR spectrum, the characteristic absorption signal of the carbonyl carbon atoms of chloranil (10a) appeared at $\delta=170.20,171.36 \mathrm{ppm}$ [29]. The ${ }^{1} \mathrm{H}-\mathrm{NMR}$ spectrum of 12a showed two broad signals at 7.68 and $8.80 \mathrm{ppm}$, due to the NH attached to the phenyl ring and the thiadiazine-NH, respectively, in addition to the phenyl protons. The IR spectrum of 12a ( $\mathrm{KBr}$ disk) showed sharp bands at 3330, 3270 and $1680 \mathrm{~cm}^{-1}$ for the secondary amino and carbonyl groups respectively. The thianthrenetetraones 13a,b exhibited absorptions at $1700-1695 \mathrm{~cm}^{-1}$ for the quinine carbonyl groups. The ${ }^{13} \mathrm{C}-\mathrm{NMR}$ spectra of 13a,b showed absorption signals around $171.36-170.86 \mathrm{ppm}$ for the chloranil or bromanil carbonyl carbon atoms. The formation of 13a,b was further confirmed by mass spectrometry. Besides the molecular ions at $416 / 412$ or $594 / 590$, the characteristic fragment ion patterns of the substituted tetrahalo compounds were observed [30]. 
Formation of these products may be rationalized by the mechanism shown in Scheme 2: an unstable CTC is formed followed by the formation of radicals $\mathbf{8}$ and $\mathbf{1 0 - H}$. Two routes could be suggested for the formation of compounds 11-13 after the recombination of the two radicals $\mathbf{8}$ and 10$\mathbf{H}$. The first one is the elimination of two molecules of $\mathrm{HCl}$ to form the intermediate 17, which splits off a molecule of substituted isothiocyanate to give the benzothiadiazine derivatives 12a-c. The second route is the elimination of one molecule of HX to give the intermediate 18. Nucleophilic attack by the 2-thiol group on the $\mathrm{C}=\mathrm{N}$ and detachment of the HS-moiety affords the intermediate $\mathbf{1 9}$ along with thiadiazoles 11a-c. The tetrahalothianthrenetetraones 13a,b could be formed via the intermediates 19 and 20 (Scheme 2).

\section{Scheme 2}

$\mathbf { 8 } + \mathbf { 1 0 } \rightleftharpoons [ \text { CT-Complex } ] \rightleftharpoons \longdiv { \mathbf { 8 } ^ { + } + 1 0 ^ { - } } \stackrel { \stackrel { + } { \mathbf { 8 } ^ { + } } \stackrel { \oplus } { \longrightarrow } } { \longrightarrow } \mathbf { 8 } ^ { \bullet }$

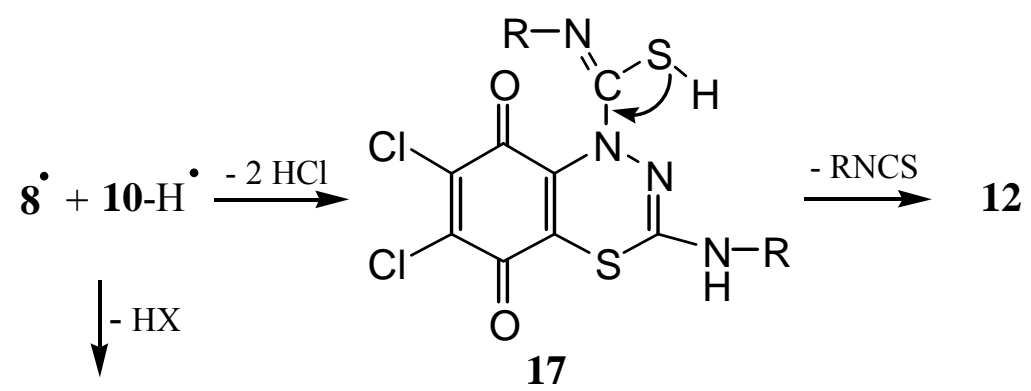

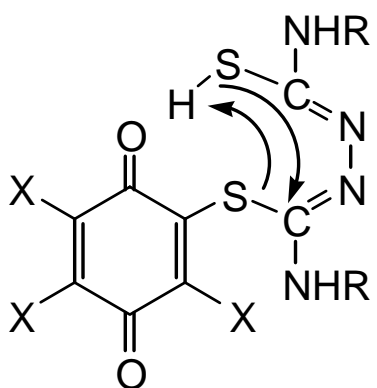

18<smiles>[X]C1=C([X])C(=O)C(S)=C([X])C1=O</smiles>

19<smiles>[X]C1=C([X])C(=O)C(S)=C(S)C1=O</smiles>

20

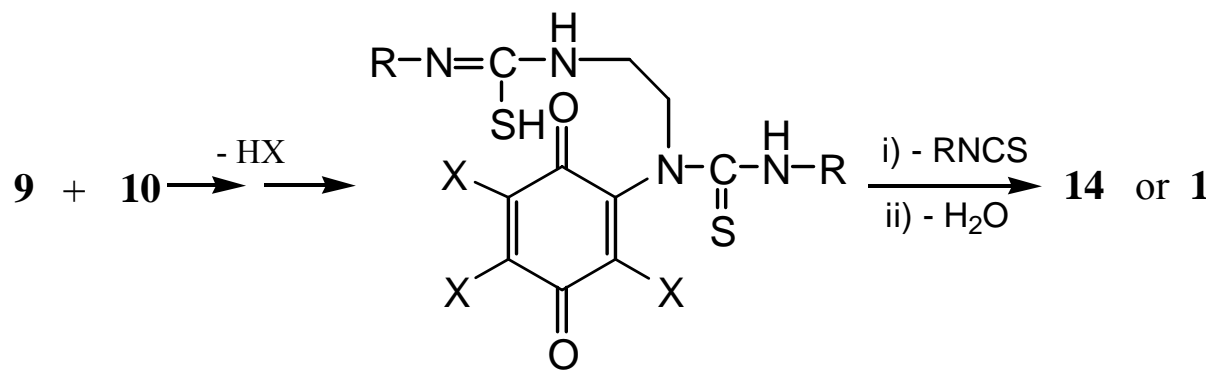


It has been reported that ethylenediamine upon reaction with allylisothiocyanate furnishes a linear thiourea, which in turn is cyclized to a bisthiazoline [31]. The present work was also undertaken to examine the reactions of 9a-c with 10a,b. Thus, two equivalents of thioureidoethylthiourea derivatives 9a-c reacted with 10a,b in THF at room temperature to afford substituted imino-[1,3,6]-thiadiazepane2-thiones 16a-c as minor (14-19\%) and trihalo-7-oxo-quinoxaline-1-carbothioic acid substituted amides 14a-c/15a-c as major products (41-49\%), in addition to the corresponding dihydrobenzoquinone derivatives. The structures of 14a-c and 15a-c were confirmed on the basis of elemental analyses, mass spectra, ${ }^{1} \mathrm{H}$ - and ${ }^{13} \mathrm{C}-\mathrm{NMR}$ data. The IR spectra of 14a-c/15a-c showed characteristic absorption bands for the secondary-NH between 3330 and $3310 \mathrm{~cm}^{-1}$ and between $1690-1680 \mathrm{~cm}^{-1}$ for the $\mathrm{C}=\mathrm{O}$ groups. The ${ }^{1} \mathrm{H}-\mathrm{NMR}$ spectrum of $14 \mathrm{a}$ shows the resonances of the methylene protons at $\mathrm{C} 3$ and $\mathrm{C} 2$ in the $\delta=3.46-3.60$ and $3.64-3.87$ ppm range, respectively. The presence of methylene groups is also evident from the ${ }^{13} \mathrm{C}$-DEPT-NMR spectrum, which exhibits negative signals at $\delta=48.77$ and $55.33 \mathrm{ppm}$. In addition, the ${ }^{1} \mathrm{H}-\mathrm{NMR}$ spectrum exhibited a broad singlet centered at $9.69 \mathrm{ppm}$ due to the $\mathrm{NH}$-attached to phenyl and $\mathrm{C}=\mathrm{S}$ groups. The decoupled carbon spectrum of 14a showed signals at $\delta=170.17$ and $180.34 \mathrm{ppm}$, assigned to $\mathrm{C}=\mathrm{O}$ and $\mathrm{C}=\mathrm{S}$, respectively [30,32].

The formation of quinoxaline products 14 and $\mathbf{1 5}$ may be rationalized through the successive substitution of one chlorine atom and elimination of a molecule of substituted isothiocyanate followed by cyclization via a condensation reaction (Scheme 2).

\section{Acknowledgements}

A. A. Hassan is indebted to the A. v. Humboldt-Foundation for the award of a fellowship from August 2003 to September 2003 and also for the donation of the Shimadzu 408 IR as well as PerkinElmer Lambda 2 Spectrophotometers.

\section{Experimental}

\section{General}

All the melting points were determined in open glass capillaries on a Gallenkamp melting point apparatus and are uncorrected. The IR spectra were recorded with a Shimadzu 408 or Bruker Vector 22 FT-IR spectrophotometers using potassium bromide pellets. A Bruker WM 300 spectrometer was used to determine ${ }^{1} \mathrm{H}-(300.13 \mathrm{MHz})$ and ${ }^{13} \mathrm{C}-(75.47 \mathrm{MHz})$ NMR spectra. Assignment of carbon resonances have been supported by DEPT experiments. Mass spectra were obtained with a Varian MAT 311 doubly focusing instrument using electron impact ionization $(70 \mathrm{eV})$. Elemental analyses were determined at the Microanalytical Center, Cairo University, Egypt. UV/Vis spectra were recorded on a Perkin-Elmer Lambda 2-spectrophotometer equipped with a thermostated cell. Preparative thin layer chromatography (plc) was carried out on $1 \mathrm{~mm}$ thick layers of silica gel slurry (Merck $\mathrm{Pf}_{254}$ ) applied on $48 \mathrm{~cm}$ wide $\mathrm{x} 20 \mathrm{~cm}$ high glass plates using the solvents mentioned below. Zones were detected by quenching of fluorescence upon exposure to $254 \mathrm{~nm}$ light and the compounds were extracted from the plates with acetone. 
Starting materials

Chloranil (2,3,5,6-Tetrachloro-1,4-benzoquinone, 10a) and bromanil (2,3,5,6-tetrabromo-1,4benzoquinone, 10b) were used as received from Aldrich. $N, N$-disubstituted hydrazinecarbothioamides 8a-c and substituted thioureidoethylthioureas 9a-c were prepared according to the literature procedures [31,33-37].

Reactions of 8a-c with chloranil (10a) and bromanil (10b).

A solution of 8a-c $(2.0 \mathrm{mmol})$ in anhydrous THF $(20 \mathrm{~mL})$ was added dropwise with stirring to a solution of chloranil (10a) or bromanil (10b) $(1.0 \mathrm{mmol})$ in the same solvent $(20 \mathrm{~mL})$. The colour of the reaction changed gradually from deep green to a blue colour. Stirring was continued for 48 hours with admission of air to complete the reaction. The reaction mixture was filtered and the blue precipitate was washed several times with cold THF and identified as the tetrahalothianthrenetetraones 13a,b. The filtrate was concentrated in vacuum and the residue separated by plc using cyclohexane/ethyl acetate $(2: 1)$ mixture into three zones. The fastest moving zone contained the thiadiazoles 11a-c, the second zone, compounds 12a-c and the slowest migrating zone contained the dihdrobenzoquinones $\mathbf{1 4}-\mathrm{H}_{2}$ or $\mathbf{1 5}-\mathrm{H}_{2}$. The zones were extracted with acetone.

$N, N^{`}$-Diphenyl-[1,3,4]thiadiazole-2,5-diamine (11a). Yield (80 mg, $15 \%$ in case of 10a and $139 \mathrm{mg}$, $26 \%$ in case of 10b), colourless crystals from DMF, m.p. $239-241{ }^{\circ} \mathrm{C}$ (lit. [38] 240-243 ${ }^{\circ} \mathrm{C}$ ).

$N, N^{`}$-Dibenzyl-[1,3,4]thiadiazole-2,5-diamine (11b). Yield (71 mg, $12 \%$ in case of 10a and $124 \mathrm{mg}$, $21 \%$ in case of $\mathbf{1 0 b}$ ), colourless crystals from methanol, m. p. $250-252{ }^{\circ} \mathrm{C}$ (lit. [34] $251{ }^{\circ} \mathrm{C}$ ).

$N, N^{`}$-Diallyl-[1,3,4]thiadiazole-2,5-diamine (11c). Yield (55 mg, $14 \%$ in case of 10a and $90 \mathrm{mg}, 23$ $\%$ in case of 10b), colourless crystals from ethanol, m.p. $133-135^{\circ} \mathrm{C}$ (lit. [36,37] $\left.135{ }^{\circ} \mathrm{C}\right)$.

3-Phenylamino-6,7-dichloro-1H-benzo[1,3,4]thiadiazine-5,8-dione (12a). Orange crystals from acetonitrile, m.p. 277-179 ${ }^{\circ} \mathrm{C}$, Yield $190 \mathrm{mg}(28 \%)$; IR cm${ }^{-1}: 3330,3270(\mathrm{NH}), 1680(\mathrm{C}=\mathrm{O}), 1620$ $(\mathrm{C}=\mathrm{N}), 1590(\mathrm{Ar}-\mathrm{C}=\mathrm{C}) ;{ }^{1} \mathrm{H}-\mathrm{NMR}(\delta): 7.11-7.32(\mathrm{~m}, 5 \mathrm{H}, \mathrm{Ph}), 7.80(\mathrm{br}, \mathrm{s}, 1 \mathrm{H}$, thiadiazine-NH), 8.68 (br, s, 1H, NHPh); ${ }^{13} \mathrm{C}-\mathrm{NMR}(\delta):$ 125.11, 128.56, 129.32 (Ph-CH), 142.43 (q-C), 127.00 (C-4a), 139.16, 141.22 (C-6,7), 152.16 (C-3), 155.63 (C-8a), 170.20, 171.36 (C-5,8); EI-MS m/z (\%): 341/339 (M+, 9), 303 (8), 267 (14), 132 (52), 104 (31), 91 (100), 77 (76), 65 (44); Anal. Calcd. for $\mathrm{C}_{13} \mathrm{H}_{7} \mathrm{Cl}_{2} \mathrm{~N}_{3} \mathrm{O}_{2} \mathrm{~S}$ (340.19): C, 45.90; H, 2.07; Cl, 20.84; N, 12.35; S, 9.43. Found: C, 46.06; H, 1.93; Cl, 20.69; N, 12.48; $\mathrm{S}, 9.56$.

3-Benzylamino-6,7-dichloro-1H-benzo[1,3,4]thiadiazine-5,8-dione (12b). Orange crystals from methanol, m.p. 291-293 ${ }^{\circ} \mathrm{C}$, Yield $177 \mathrm{mg}(25 \%)$; IR cm${ }^{-1}: 3340,3255(\mathrm{NH}), 1675(\mathrm{C}=\mathrm{O}), 1630$ $(\mathrm{C}=\mathrm{N}), 1585(\mathrm{Ar}-\mathrm{C}=\mathrm{C}) ;{ }^{1} \mathrm{H}-\mathrm{NMR}(\delta): 4.64\left(\mathrm{br}, \mathrm{s}, 2 \mathrm{H}, \mathrm{CH}_{2} \mathrm{Ph}\right), 7.06-7.24(\mathrm{~m}, 5 \mathrm{H}, \mathrm{Ph}), 7.70$ (br, s, 1H, thiadiazine-NH), $8.43\left(\mathrm{br}, \mathrm{s}, 1 \mathrm{H}, \mathrm{NHCH}_{2} \mathrm{Ph}\right) ;{ }^{13} \mathrm{C}-\mathrm{NMR}(\delta): 47.94\left(\mathrm{CH}_{2} \mathrm{Ph}\right), 126.56,127.18$, 128.41(Ph-CH), 141.42 (q-C), 127.24 (C-4a), 139.36, 141.11 (C-6,7), 151.83 (C-3), 155.42 (C-8a), 169.96, 170.83 (C-5,8); EI-MS m/z (\%): 355/353 (M+1 11), 317 (5), 281 (8), 104 (27), 91 (83), 71 
(100); Anal. Calcd. for $\mathrm{C}_{14} \mathrm{H}_{9} \mathrm{Cl}_{2} \mathrm{~N}_{3} \mathrm{O}_{2} \mathrm{~S}$ (354.22): C, 47.47; H, 2.56; Cl, 20.02; N, 11.86; S, 9.05. Found C, 47.31; H, 2.73; Cl, 19.88; N, 11.98; S, 8.93.

3-Allylamino-6,7-dichloro-1H-benzo[1,3,4]thiadiazine-5,8-dione (12c). Pale orange crystals from methanol, m.p. 189-199 ${ }^{\circ} \mathrm{C}$, Yield $134 \mathrm{mg}(22 \%)$; IR cm${ }^{-1}: 3340,3260(\mathrm{NH}), 2960,2840$ (Ali-CH), $1685(\mathrm{C}=\mathrm{O}), 1610(\mathrm{C}=\mathrm{N}) ;{ }^{1} \mathrm{H}-\mathrm{NMR}(\delta): 4.22\left(\mathrm{~m}, 2 \mathrm{H}\right.$, allyl- $\left.\mathrm{CH}_{2} \mathrm{~N}\right)$, 5.14-5.17 (m, 2H, allyl- $\left.\mathrm{CH}_{2}=\right)$, 5.92-6.04 (m, 1H, allyl-CH=), 7.54 (br, s, 1H, allyl-NH), 7.86 (br, s, $1 \mathrm{H}$, thiadiazine-NH); ${ }^{13} \mathrm{C}-\mathrm{NMR}$

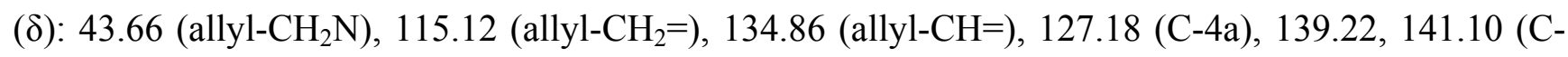
6,7), 151.36 (C-3), 154.76 (C-8a), 170.76, 171.48 (C-5,8); EI-MS m/z (\%): 305/303 (M+, 21), 267 (14), 231 (9), 203 (11), 99 (100), 41 (61); Anal. Calcd. for $\mathrm{C}_{10} \mathrm{H}_{7} \mathrm{Cl}_{2} \mathrm{~N}_{3} \mathrm{O}_{2} \mathrm{~S}$ (304.16): C, 39.49; H, 2.32; Cl, 23.31; N, 13.82; S, 10.54. Found C, 39.35; H, 2.24; Cl, 23.51; N, 14.01; S, 10.34 .

2,3,7,8-Tetrachlorothianthrene-1,4,6,9-tetraone (13a). Blue crystals from DMF, m.p. 342-344 ${ }^{\circ} \mathrm{C}$, Yield $170 \mathrm{mg}$ (41\%); IR cm ${ }^{-1}: 1695(\mathrm{C}=\mathrm{O})$; ${ }^{13} \mathrm{C}-\mathrm{NMR}(\delta): 143.47(\mathrm{C}-2,3,7,8), 149.32$ (C4a,5a,9a,10a), 171.36 (C-1,4,6,9); EI-MS m/z (\%): 416/412 (M+, 100), 398 (39), 379 (12), 349 (16), 321 (19), 115 (55), 87 (91), 64 (36), 36 (69); Anal. Calcd. for $\mathrm{C}_{12} \mathrm{Cl}_{4} \mathrm{O}_{4} \mathrm{~S}_{2}$ (414.07): C, 34.81; $\mathrm{Cl}$, 34.25; S, 15.49. Found C, 34.66; Cl, 34.41; S, 15.63.

2,3,7,8-Tetrabromothianthrene-1,4,6,9-tetraone (13b). Blue crystals from DMF, m.p. $>360{ }^{\circ} \mathrm{C}$, Yield $260 \mathrm{mg}$ (44\%); IR cm ${ }^{-1}: 1700(\mathrm{C}=\mathrm{O}) .{ }^{13} \mathrm{C}-\mathrm{NMR}(\delta): 138.16$ (C-2,3,7,8), 149.11 (C-4a,5a,9a,10a), 170.86 (C-1,4,6,9); EI-MS m/z (\%): 594/590 (M+1, 100), 512 (20), 496 (26), 416 (18), 260 (66), 188 (56), 142 (33), 116 (83), 60 (54); Anal. Calcd. for $\mathrm{C}_{12} \mathrm{Br}_{4} \mathrm{O}_{4} \mathrm{~S}_{2}$ (591.87): C, 24.35; Br, 54.00; S, 10.84; found $\mathrm{C}, 24.51 ; \mathrm{Br}, 53.86 ; \mathrm{S}, 11.02$.

Reactions of 9a-c with chloranil (10a) and bromanil (10b).

A solution of 9a-c $(1.0 \mathrm{mmol})$ in anhydrous THF $(15 \mathrm{~mL})$ was added dropwise with stirring to a solution of 10a,b $(1.0 \mathrm{mmol})$ in anhydrous THF $(20 \mathrm{~mL})$. The mixture was heated under reflux for 5 hours, during which it turned from yellow into reddish orange. The mixture was concentrated under vacuum and the residue separated by plc using cyclohexane/ethyl acetate (3:1) as developing solvent to give numerous coloured zones, three of which (with the highest intensity) were extracted and removed. The fastest migrating one, which quenched all indicator fluorescence upon exposure to $254 \mathrm{~nm} \mathrm{UV}$ light, contained the thiadiazepanes 16a-c, the second zone (which was always characterized by an orange colour) contained the quinoxalines 14a-c and 15a-c, while the third zone contained the dihydrobenzoquinones $14-\mathrm{H}_{2}$ and $15-\mathrm{H}_{2}$.

5,6,8-Trichloro-7-oxo-3,7-dihydro-2H-quinoxaline-1-carbothioic acid phenyl amide (14a). Brown crystals from ethanol, m.p. 254-256 ${ }^{\circ} \mathrm{C}$, Yield $189 \mathrm{mg}(49 \%)$; IR cm ${ }^{-1}$ : 3325 (NH), 2965 (Ali-CH), $1685(\mathrm{C}=\mathrm{O}), 1590(\mathrm{Ar}-\mathrm{C}=\mathrm{C})$; ${ }^{1} \mathrm{H}-\mathrm{NMR}(\delta): 3.46-3.60\left(\mathrm{~m}, 2 \mathrm{H}\right.$, quinoxaline-3- $\left.\mathrm{H}_{2}\right), 3.64-3.87(\mathrm{~m}, 2 \mathrm{H}$, quinoxaline-2- $\left.\mathrm{H}_{2}\right), 7.08-7.37(\mathrm{~m}, 5 \mathrm{H}, \mathrm{Ph}), 9.69$ (br, s, $\left.1 \mathrm{H}, \mathrm{NHPh}\right) ;{ }^{13} \mathrm{C}-\mathrm{NMR}(\delta): 48.77,55.33$ (quinoxaline-C-3,2), $120.11(\mathrm{C}-8), 124.83,125.31,128.86(\mathrm{Ph}-\mathrm{CH}), 139.41(\mathrm{q}-\mathrm{C}), 138.46,141.33$ (C5,6), 151.12 (C-4b), 158.36 (C-4a), 170.17 (C-7), 180.34 (C=S); EI-MS m/z (\%): 387/383 (M+, 36), 349 (11), 277 (8), 221 (21), 205 (9), 135 (57), 91 (100), 77 (81), 65 (64); Anal. Calcd. for 
$\mathrm{C}_{15} \mathrm{H}_{10} \mathrm{Cl}_{3} \mathrm{~N}_{3} \mathrm{OS}$ (386.68): C, 46.59; H, 2.61; Cl, 27.51; N, 10.87; S, 8.29. Found C, 46.68; H, 2.53; $\mathrm{Cl}$, 27.38 ; N, 11.03; S, 8.44.

5,6,8-Trichloro-7-oxo-3,7-dihydro-2H-quinoxaline-1-carbothioic acid benzyl amide (14b). Brown crystals from acetonitrile, m.p. 269-271 ${ }^{\circ} \mathrm{C}$, Yield $188 \mathrm{mg}(47 \%)$; IR cm${ }^{-1}: 3320(\mathrm{NH}), 2960,2870$ (Ali-CH), $1690(\mathrm{C}=\mathrm{O}), 1600(\mathrm{Ar}-\mathrm{C}=\mathrm{C}) ;{ }^{1} \mathrm{H}-\mathrm{NMR}(\delta): 3.50-3.61\left(\mathrm{~m}, 2 \mathrm{H}\right.$, quinoxaline-3- $\left.\mathrm{H}_{2}\right), 3.70-3.85$ (m, 2H, quinoxaline-2- $\mathrm{H}_{2}$ ), $4.60\left(\mathrm{br}, \mathrm{s}, 2 \mathrm{H}, \mathrm{CH}_{2} \mathrm{Ph}\right)$ 7.0-7.29 (m, 5H, Ph), 9.42 (br, s, $1 \mathrm{H}, \mathrm{NHCH}_{2} \mathrm{Ph}$ ); ${ }^{13} \mathrm{C}-\mathrm{NMR}(\delta): 48.68,55.19$ (quinoxaline-C-3,2), $50.24\left(\mathrm{CH}_{2} \mathrm{Ph}\right), 119.82(\mathrm{C}-8), 126.52,127.14,128.95$ (Ph-CH), 140.13 (q-C), 138.37, 141.20 (C-5,6), 150.76 (C-4b), 158.82 (C-4a), 169.93 (C-7), 181.12 $(\mathrm{C}=\mathrm{S}) ; \mathrm{EI}-\mathrm{MS} \mathrm{m} / \mathrm{z}$ (\%): 400/397 (M+, 22), 363 (17), 263 (27), 235 (11), 149 (42), 91 (62), 77 (100), 65 (83); Anal. Calcd. for $\mathrm{C}_{16} \mathrm{H}_{12} \mathrm{Cl}_{3} \mathrm{~N}_{3} \mathrm{OS}$ (400.71): C, 47.96; H, 3.02; Cl, 26.54; N, 10.49; S, 8.00. Found C, 48.12; H, 2.96; Cl, 26.39; N, 10.66; S, 7.86.

5,6,8-Trichloro-7-oxo-3,7-dihydro-2H-quinoxaline-1-carbothioic acid allyl amide (14c). Pale brown crystals from ethanol, m.p. 167-169 ${ }^{\circ} \mathrm{C}$, Yield $154 \mathrm{mg}(44 \%)$; IR cm${ }^{-1}: 3330(\mathrm{NH}), 2970,2890$ (Ali$\mathrm{CH}), 1685(\mathrm{C}=\mathrm{O})$; ${ }^{1} \mathrm{H}-\mathrm{NMR}\left((\delta)\right.$ : 3.48-3.57 (m, 2H, quinoxaline-3- $\left.\mathrm{H}_{2}\right), 3.86-3.86(\mathrm{~m}, 2 \mathrm{H}$, quinoxaline2- $\left.\mathrm{H}_{2}\right), 4.22\left(\mathrm{~m}, 2 \mathrm{H}\right.$, allyl- $\left.\mathrm{CH}_{2} \mathrm{~N}\right), 5.17-5.20\left(\mathrm{~m}, 2 \mathrm{H}\right.$, allyl- $\left.\mathrm{CH}_{2}=\right), 5.84-5.92(\mathrm{~m}, 1 \mathrm{H}$, allyl-CH=), 7.54 (br, s, $1 \mathrm{H}$, allyl-NH); ${ }^{13} \mathrm{C}-\mathrm{NMR}(\delta): 43.62\left(\right.$ allyl- $\left.\mathrm{CH}_{2} \mathrm{~N}\right), 48.61,55.12$ (quinoxaline-C-3,2), 114.96 (allyl- $\mathrm{CH}_{2}=$ ), 119.86 (C-8), 134.76 (allyl-CH=), 138.56141 .13 (C-5,6), 151.10 (C-4b), 158.63 (C-4a), 170.12 (C-7), 180.66 (C=S); EI-MS m/z (\%): 351/347 (M+, 32), 313 (18), 277 (6), 241 (11), 185 (24), 99 (76), 41 (100), 36 (54); Anal. Calcd. for $\mathrm{C}_{12} \mathrm{H}_{10} \mathrm{Cl}_{3} \mathrm{~N}_{3} \mathrm{OS}$ (350.65): C, 41.10; H, 2.87; Cl, 30.33; N, 11.98; S, 9.14. Found C, 41.26; H, 2.69; Cl, 30.13; N, 12.11; S, 9.26.

5,6,8-Tribromo-7-oxo-3,7-dihydro-2H-quinoxaline-1-carbothioic acid phenyl amide (15a). Reddishbrown crystals from ethanol, m.p. 273-275 ${ }^{\circ} \mathrm{C}$, Yield $239 \mathrm{mg}(46 \%)$; IR cm${ }^{-1}: 3310$ (NH), 2960 (Ali$\mathrm{CH}), 1680(\mathrm{C}=\mathrm{O}), 1600(\mathrm{Ar}-\mathrm{C}=\mathrm{C}) ;{ }^{1} \mathrm{H}-\mathrm{NMR}(\delta)$ : 3.44-3.61 (m, 2H, quinoxaline-3- $\left.\mathrm{H}_{2}\right)$, 3.66-3.85 (m, $2 \mathrm{H}$, quinoxaline-2- $\left.\mathrm{H}_{2}\right), 7.10-7.35(\mathrm{~m}, 5 \mathrm{H}, \mathrm{Ph}), 9.65$ (br, s, $\left.1 \mathrm{H}, \mathrm{NHPh}\right) ;{ }^{13} \mathrm{C}-\mathrm{NMR}(\delta): 48.76,55.12$ (quinoxaline-C-3,2), 103.34 (C-8), 124.34, 125.16, 128.83 (Ph-CH), 139.42 (q-C), 127.66, 130.18 (C5,6), 150.66 (C-4b), 158.36 (C-4a), 170.10 (C-7), 180.36 (C=S); EI-MS m/z (\%): 519/515 (M , 18), 489 (12), 461 (14), 437 (21), 357 (18), 277 (12), 142 (38), 91 (67), 77 (83), 65 (100); Anal. Calcd. For $\mathrm{C}_{15} \mathrm{H}_{10} \mathrm{Br}_{3} \mathrm{~N}_{3} \mathrm{OS}$ (520.04): C, 34.64; H, 1.94; Br, 46.10; N, 8.08; S, 6.17. Found C, 34.51; H, 2.12; Br, $45.93 ; \mathrm{N}, 7.96 ; \mathrm{S}, 6.29$.

5,6,8-Tribromo-7-oxo-3,7-dihydro-2H-quinoxaline-1-carbothioic acid benzyl amide (15b). Reddishbrown crystals from acetonitrile, m.p. 282-284 ${ }^{\circ} \mathrm{C}$, Yield $219 \mathrm{mg}(41 \%)$; IR cm ${ }^{-1}: 3330(\mathrm{NH}), 2965$, $2840\left(\right.$ Ali-CH), $1690(\mathrm{C}=\mathrm{O}), 1590(\mathrm{Ar}-\mathrm{C}=\mathrm{C})$; ${ }^{1} \mathrm{H}-\mathrm{NMR}(\delta)$ : 3.53-3.64 (m, 2H, quinoxaline-3- $\left.\mathrm{H}_{2}\right)$, 3.68-3.83 (m, 2H, quinoxaline-2- $\left.\mathrm{H}_{2}\right), 4.64$ (br, s, 2H, $\left.\mathrm{CH}_{2} \mathrm{Ph}\right), 6.98-7.28(\mathrm{~m}, 5 \mathrm{H}, \mathrm{Ph}), 9.45$ (br, s, $1 \mathrm{H}$, $\mathrm{NHCH}_{2} \mathrm{Ph}$ ); EI-MS m/z (\%): 533/529 (M+18), 503 (11), 474 (6), 451 (27), 371 (18), 291 (16), 142 (36), 91 (100), 77 (67), 65 (43): Anal. Calcd. for $\mathrm{C}_{16} \mathrm{H}_{12} \mathrm{Br}_{3} \mathrm{~N}_{3} \mathrm{OS}$ (534.06): C, 35.98; H, 2.26; $\mathrm{Br}$, 44.88; N, 7.87; S, 6.00. Found C, 36.14; H, 2.18; Br, 45.08; N, 7.96; S, 5.87.

5,6,8-Tribromo-7-oxo-3,7-dihydro-2H-quinoxaline-1-carbothioic acid allyl amide (15c). Reddish brown crystals from ethanol, m.p. $185-187^{\circ} \mathrm{C}$, Yield $208 \mathrm{mg}$ (43\%); IR cm ${ }^{-1}: 3310$ (NH), 2970, 2890 
(Ali-CH), $1685(\mathrm{C}=\mathrm{O})$; ${ }^{1} \mathrm{H}-\mathrm{NMR}(\delta)$ : 3.46-3.58 (m, 2H, quinoxaline-3- $\left.\mathrm{H}_{2}\right), 3.62-3.78(\mathrm{~m}, 2 \mathrm{H}$, quinoxaline-2- $\left.\mathrm{H}_{2}\right), 4.18\left(\mathrm{~m}, 2 \mathrm{H}\right.$, allyl- $\left.\mathrm{CH}_{2} \mathrm{~N}\right), 5.18-5.22\left(\mathrm{~m}, 2 \mathrm{H}\right.$, allyl- $\left.\mathrm{CH}_{2}=\right), 5.92-6.03(\mathrm{~m}, 1 \mathrm{H}$, allyl$\mathrm{CH}=$ ), 7.58 (br, s, 1H, allyl-NH); EI-MS m/z (\%): 483/479 (M+, 21), 401 (16), 321 (11), 241 (6), 213 (17), 185 (32), 86 (53), 41 (100); Anal. Calcd. for $\mathrm{C}_{12} \mathrm{H}_{10} \mathrm{Br}_{3} \mathrm{~N}_{3} \mathrm{OS}$ (484.01); C, 29.78; H, 2.08; Br, 49.53; N, 8.68; S, 6.63. Found C, 29.64; H, 1.96; Br, 49.68; N, 8.52; S, 6.47.

7-Phenylimino-[1,3,6]thiadiazepane-3-thione (16a). Yield (45 mg, $19 \%$ ), colourless crystals from methanol, m.p. $233-235^{\circ} \mathrm{C}$ (lit. [37] 235-237 ${ }^{\circ} \mathrm{C}$ ).

7-Benzylimino-[1,3,6]thiadiazepane-3-thione (16b). Yield (40 mg, $16 \%$ ), colourless crystals from ethanol, m.p. $130-132{ }^{\circ} \mathrm{C}$ (lit. [37] 128-130 ${ }^{\circ} \mathrm{C}$ ).

7-Allylimino-[1,3,6]thiadiazepane-3-thione (16c). Yield (28 mg, $14 \%$ ), colourless crystals from ethanol, m.p. $100-101^{\circ} \mathrm{C}$ (lit. [37] 98-100 ${ }^{\circ} \mathrm{C}$ ).

\section{References}

1. Patai, S.; Rappoport Z. "The Chemistry of Quinonoid Compounds"; Wiley Interscience: New York, 1988, vol. 2, part 1, pp. 552- 570.

2. Billman, J. H.; Thomas, D. G.; Barnes, D. K. 2,5-Diamino-1,4-benzoquinone. J. Am. Chem. Soc. 1946, 68, 2103-2103.

3. Kouno, K.; Ogawa, C.; Shimoura, Y.; Yano, H.; Veda, Y. Interaction of Imidazole Derivatives with Electron Acceptors. II. Reaction Products of Imidazole with p-Benzoquinones. Chem. Pharm. Bull. 1981, 29, 301-7.

4. Ballesteros, P.; Claramunt, R. M.; Escolastico, C.; Santa Maria, M. D. Reaction of Pyrazole Addition to Quinones. J. Org. Chem. 1993, 57, 1873-76.

5. Foster, R.; Kulevsky, N.; Wanigasekera, D. S. Reaction of Amino-acids with p-Benzoquinones. $J$. Chem. Soc. Perkin Trans. 1. 1974, 1318-1321.

6. Khan, A. H.; Driscoll, J. S. Potential Central Nervous System Antitumor Agents. Aziridinylbenzoquinones 1. J. Med. Chem. 1976, 19, 313-317.

7. Chan, F.; Khan, A. H.; Driscoll, J. S., Potential Central Nervous System Antitumor Agents. Aziridinylbenzoquinones 2. J. Med. Chem. 1976, 19, 1302-1308.

8. Hassan, A. A.; Mohamed, N. K.; Aly, A. A.; Mourad, A. E. A new Synthesis of Quinoxaline, Imidazolidine, Indole, Carbazole Derivatives and their Antibacterial Activity. Pharmazie, 1997, 52, 282-287.

9. Hassan, A. A.; Mohamed, N. K.; Ibrahim, Y. A.; Mourad, A. E. Chemical Interactions of Thiosemicarbazides with 1,4-Benzo- and 1,4-Naphthoquinones. Liebigs Ann. Chem. 1993, 695697.

10. Ibrahim, Y. A.; Hassan, A. A.; Mohamed, N. K.; Mourad, A. E. 3-Amino-4-arylazopyrazole: CT-complexation and a Novel Synthesis of Pyrazolo[2,3-a]quinazoline. Arch. Pharm. (Weinheim) 1992, 325, 389-392.

11. Hassan, A. A.; Mohamed, N. K.; Aly, A. A.; Mourad, A. E. Reaction of 3,5-Diamino-4arylazopyrazoles with Chlorinated Quinones. Bull. Soc. Chim. Belg. 1996, 105, 159-162. 
12. Hassan, A. A.; Mohamed, N. K.; Aly, A. A.; Mourad, A. E. A Novel Synthesis of 1,2,4Triazolo[1,5-a]isoindolinetrione, 1,2,4-Triazolo[1,5-a]pyrimidine and 1,2,4-Triazolo[2,3-a]quinazolinedione Derivatives and Their Antibacterial Activity. Pharmazie, 1997, 52, 23-28.

13. Hassan, A. A.; Mohamed, N. K.; Ibrahim, Y. A.; Sadak, K. U.; Mourad, A. E. Chemical Interaction Between 1,2,4-Triazole-3-thiols and $\pi$-Acceptors. Bull. Chem. Soc. Jpn. 1993, 66, 2612-2626.

14. Hammam, A. S.; Bayoumy, B. E. Heterocyclic Quinines. XIII. Reaction of Thioamides With 2,3Dichloro-1,4-naphthoquinone. A Novel Synthesis of Naphtha[2,3-d]thiazole-4,9-diones. Collect. Czech. Chem. Commun. 1985, 50, 71-9.

15. Katritzky, A. R.; Fan, W. Q. Some Novel Quinone-Type Dyes Containing Naphthoquinone and Related Fused Ring Systems. J. Heterocyclic Chem. 1988, 25, 901-906.

16. Katritzky, A. R.; Fan, W. Q.; Linand, Q. I.; Bayyuk, S. Novel Chromophoric Heterocycles Based on Maleimide and Naphthoquinone. J. Heterocyclic Chem. 1989, 26, 885.

17. Matsuoka, M.; Iwamoto, A.; Furukawa, N.; Kitao, T. Reaction of 2,3-Dicloro-1,4-naphthoquinone With Dithiooxamide. Synthesis of Dibenzo[b,i]thianthrene-5,7,12,14-tetrone. J. Heterocyclic Chem. 1991, 28, 1445-1447.

18. Matsuoka, M.; Iwamoto, A.; Furukawa, N.; Kitao, T. Syntheses of Polycyclic-1,4-dithiines and Related Heterocycles. J. Heterocyclic Chem. 1992, 29, 439-445.

19. Matsuoka, M.; Iwamoto, A. The Structure of The CT Complex Between 5,7,12,14Tetramethoxydibenzo[b,i]thianthrene with TCNQ. J. Heterocyclic Chem. 1993, 30, 173-176.

20. Katritzky, A. R.; Fan, W. Q. A Reexamination of The Reaction of 2,3-Dichloro-1,4naphthoquinone With Thioamides, J. Heterocyclic Chem. 1993, 30, 1679-1681

21. Raphael, E.; Joshua, C. P.; Koshy, L. Alkali Catalyzed Thermal Cyclization of 1-Substituted and 1,6-Disubstituted 2,5-dithiobiureas: Formation of 1,2,5-Dithiones and/ or 1,3,4-Thiadiazoline-5thiones. Indian J. Chem. 1989, 28B, 635-638.

22. Kurzer, F.; Secker, J. L. Addition-Cyclization of Ethoxycarbonyl Isothiocyanate With Hydrazine Derivatives as a Source of Thiadiazoles and Triazoles. J. Heterocyclic Chem., 1989, 26, 355-359.

23. Tomita, Y; Kabashima, S; Okawara, T; Yamasaki, T. Furukawa, M., Heterocyclization of 2,4Disubstituted thiosemicarbazides with Haloketones. J. Heterocyclic Chem., 1990, 27, 707-710.

24. Suni, M. M.; Nair, V. A; Joshua, C. P. Heterocyclization of 1-Aryl/alkyl-2-thiobiureas to 4Aryl/alkyl-3-substituted- $\Delta^{2}$-1,2,4-triazolin-5-ones. Tetrahedron, 2001, 57, 2003-2009.

25. Dobosz, M.; Pachuta-Stec A. Synthesis of New Derivatives of 3-Benzyl- $\Delta^{2}-1,2,3$-triazoline-5thione and 5-Benzyl-1,3,4-thiadiazole. Acta Pol. Pharm. 1996, 53, 123-131.

26. Korzycka, L.; Glowka, M.; Janicka, J. Synthesis and X-ray Studies of Derivatives of 1,2,4Triazolidine-3-thiones. Pol. J. Chem. 1998, 72, 73-77.

27. Dittmer, D. C. "No-Solvent” Organic Synthesis. Chem. Ind. 1997, 19, 779-784.

28. Invidiata, F. P; Furno, G.; Lampronti, I.; Simoni, D. 1,2,4-Triazoles. Improved Synthesis of 5Substituted 4-Amino-3-mercapto-(4H)-1,2,4-triazoles and a Facile Route to 3,6-Disubstituted 1,2,4-Triazolo[3,4-b][1,2,3]thiadiazoles. J. Heterocyclic Chem., 1997, 34, 1255-1258.

29. Kalinowski, H. O.; Berger, S.; Braun, S. ${ }^{13} C-N M R$ Spectroscopy; Georg Thieme Verlag: Stuttgart, 1984.

30. Silverstein, R. M. Spectrometric Identificaation of Organic Compounds; John Wiley and Sons, Inc.; New York, 1974. 
31. Mizrakh, L. T.; Polanskaya, L. Yu.; Gvozdetskii, A. N.; Vosil'ev, A. M.; Ivanova, T. M.; Lisina, N. I. Synthesis of Some of $N$-allyl- $N^{`}$-Substituted thiocarbamide Derivatives and Study of their Antiradiation Properties, Khim-Farm Zn. 1987, 21, 322-328.

32. Reiter, J.; Barkôczy, J., On triazoles. XXXIX [1]. The Reaction of Triazolyl thiohydrazides with Isocyanates and Isothiocyanates. J. Heterocyclic Chem. 1993, 30, 333-343.

33. Kepe, V.; Pozvàn, F.; Golobič, A.; Polanc, S.; Koéevar, M. Migration of an Acyl Group in the Pyrazole System: Synthesis of 1-Acyl-3-hydroxy-1H-pyrazoles and Related Derivatives. A New Preparation of N,N`-Diacylhydrazines. J. Chem. Soc. Perkin Trans. 1, 1998, 2813-2816.

34. Heinz, E.; Berscheid, R. Preparation and Application of Thiosemicarbazide and Thiocarbazide Derivatives as Antimicrobial Agent. Sofw J. 1994, 120, 286-90; [C. A. 1996, 124, 55859 w].

35. Simiti, A. I.; Cosma, N.; Proinov, I., Addition to Isothiocyanates I. Addition of Thiosemicarbazides to Isothiocyanates. Acad. Rep. Pop. Rom., Filiala Cluj, studii cercetavdri Chim. 1957, 8, 315-333.

36. Guha, P. C. Ring Clousure of Hydrazodithio- and -Monothiodicarbonamides with acetic anhydride. J. Am. Chem. Soc. 1923, 45, 1036-1039.

37. Hassan, A. A.; Mourad, A. E.; El-Shaieb, K. M.; Abou-Zied, A. H.; Döpp, D. Thermolysis of Symmetrical Dithiobiurea and Thioureidoethylthiourea Derivatives. J. Heteroatom Chem. 2003, $14,535-541$.

38. Ried, W.; Oxenius, R. Synthesis and Pyrolysis of Symmetrical Mercapto derived. Chem. Ber. 1973, 106, 484-490.

Sample availability: Samples of compounds 2, 11, 13a and 16 are available from MDPI.

(C) 2005 by MDPI (http://www.mdpi.org). Reproduction is permitted for noncommercial purposes. 